\title{
Nonlinear Propagation of Pulsar Radiation
}

\author{
P. K. Shukla and L. Stenflo \\ Fakultät für Physik und Astronomie, Ruhr-Universität Bochum, \\ D-44780 Bochum, Germany. Email: ps@tp4.ruhr-uni-bochum.de
}

\begin{abstract}
The nonlinear propagation of pulsar radiation in an electronpositron plasma is considered. Accounting for the pulsar radiation ponderomotive force, the relativistic mass variation, and the plasma magnetization, we obtain a pair of nonlinear equations which exhibit coupling of pulsar radiation with the background plasma slow motions in magnetized plasmas. The modulational/filamentation instability as well as the localization of pulsar radiation are investigated. We conclude that our investigation is relevant to the microstructures in the pulsar magnetosphere.
\end{abstract}

\section{Introduction}

Present models (Sturrock 1971; Ruderman \& Sutherland 1975; Arons \& Barnard 1986) suggest that a strongly magnetized electron-positron plasma is formed near the polar caps of a neutron star. The investigation of the coherent radio emission from the pulsar magnetosphere has attracted a great deal of attention (Cordes 1979; Michel 1982; Asseo et al. 1990). The observed pulsar radiation exhibits microstructures (Bartel et al. 1980; Ferguson 1981). The latter can only be understood in terms of a nonlinear model.

In this paper, we consider the nonlinear propagation of coherent pulsar radiation through a magnetized electron-positron plasma. It is shown that the nonlinear effects caused by harmonic generation, relativistic particle mass variation, as well as the pulsar radiation pressure can produce an amplitude modulation of the pulsar radiation, leading to modulational instability and localized wave trains including envelope solitons. Our theoretical model is capable of producing the salient features of the pulsar microstructures.

\section{The Model}

Let us consider the nonlinear propagation of pulsar radiation through a strongly magnetized electron-positron plasma in which the mass $m$ of the plasma particles are equal. The external magnetic field $B_{0}$ is along the $z$ axis. The dispersion relation of the linearly polarized ordinary mode (O-mode) is $N^{2}=1-2 \omega_{p}^{2} / \omega^{2}$, where $N=k c / \omega$ is the refractive index, $\omega$ is the frequency, $\hat{\mathbf{x}} k$ is the wave vector, $c$ is the speed of light, and $\omega_{p}=\left(4 \pi n e^{2} / m\right)^{1 / 2}$ is the plasma frequency. The electric field vector of the $\mathrm{O}$-mode is along the $z$ axis. On the other hand, 
linearly polarized (circularly polarized) extraordinary (right or left-hand) pulsar radiation propagating across (along) the $z$ axis satisfies the dispersion relation $N^{2}=1-2 \omega_{p}^{2} /\left(\omega^{2}-\omega_{c}^{2}\right)$, where $\omega_{c}=e B_{0} / m c$ is the electron gyrofrequency. Thus, the frequency of the $\mathrm{X}$-mode and the circularly polarized radiation are given by $\omega^{2}=\left[\omega_{r}^{2} \pm\left(\omega_{r}^{4}-4 k^{2} c^{2} \omega_{c}^{2}\right)^{1 / 2}\right] / 2$, where $\omega_{r}^{2}=k^{2} c^{2}+\omega_{H}^{2}$ and $\omega_{H}^{2}=$ $2 \omega_{p}^{2}+\omega_{c}^{2}$. The frequency of the long wavelength $\left(k c<<\omega_{c}, \omega_{p}\right)$ fast mode is $\omega \approx \omega_{H}\left(1+k^{2} c^{2} \omega_{p}^{2} / \omega_{H}^{4}\right)$, whereas that of short wavelength modes $\left(k c>>\omega_{c}, \omega_{p}\right)$ is $\omega \approx k c\left(1+\omega_{p}^{2} / k^{2} c^{2}\right)$. Furthermore, the frequency of the long wavelength slow mode is given by $\omega=\left[k c /(1+\alpha)^{1 / 2}\right]\left[1-k^{2} c^{2} \omega_{p}^{2} / \omega_{c}^{4}(1+\alpha)^{2}\right]$, where $\alpha=2 \omega_{p}^{2} / \omega_{c}^{2}$.

In the following, we present the nonlinear model for the pulsar microstructures.

\subsection{The nonlinear $\mathrm{O}$-mode}

Combining the Maxwell equations and accounting for the relativistic mass variation and the harmonic generation nonlinearities, we obtain the wave equation (Shukla 1985) for the ordinary mode pulsar radiation

$$
\left(\partial_{t}^{2}-c^{2} \partial_{x}^{2}+2 \omega_{p}^{2}\right) \nu=R \omega_{p}^{2}|\nu|^{2} \nu,
$$

where $\nu=e E_{z} / m_{0} \omega c, e$ is the magnitude of the electron charge, $E_{z}$ is the component of the electric field along the $z$ axis, $m_{0}$ is the rest mass of the electrons or positrons, $\omega^{2}=2 \omega_{p}^{2}+k^{2} c^{2}$, and $R=(3 / 4)+k^{2} c^{2} /\left(\omega_{c}^{2}-4 \omega^{2}\right)$. In $R$ the first and second terms come from the relativistic mass variation and the harmonic generation associated with the nonlinear Lorentz force, respectively. Assuming that the nonlinear effects produce a slowly varying pulsar radiation envelope, Eq. (1) leads in the WKB approximation $\left(\partial_{\tau} E_{z}<<\omega E_{z}\right)$ to

$$
i\left(\partial_{\tau}+v_{g} \partial_{x}\right) \nu+P \partial_{x}^{2} \nu+Q|\nu|^{2} \nu=0,
$$

where $v_{g}=k c^{2} / \omega$ is the group velocity of the pulsar radiation, $P=c^{2} / 2 \omega$, and $Q=R \omega_{p}^{2} / 2 \omega$.

The modulational instability of a constant amplitude $\left(E_{0 z}\right)$ pulsar pump can be investigated from the nonlinear dispersion relation

$$
\left(\Omega-K v_{g}\right)^{2}-\frac{K^{4} c^{4}}{4 \omega^{2}}+\frac{K^{2} c^{2}}{2 \omega^{2}} \omega_{p}^{2} \nu_{0}^{2} R=0,
$$

where $\Omega(<<\omega)$ and $K$ are the frequency and wavenumber of non-resonant perturbations modulating the pulsar radiation and $\nu_{0}=\nu\left(E_{0 z}\right)$. The modulations are unstable if $\nu_{0}>K c / \sqrt{2 R} \omega_{p}$, with $R>0$. The maximum growth rate is $\gamma_{m}=\omega_{p}^{2} R \nu_{0}^{2} / 2 \omega$. A possible final state of the modulational instability can be represented as an envelope soliton

$$
\nu^{2}=\nu_{m}^{2} \operatorname{sech}^{2}(\xi / \delta),
$$

where $\xi=x-v_{g} \tau, \nu_{m}=\nu\left(E_{z m}\right), E_{z m}$ is the maximum amplitude at $\xi=0$, and $\delta=(2 / R)^{1 / 2} c / \omega_{p} \nu_{m}$ is the pulse width. The temporal pulse width is roughly $\tau_{0} \sim 1 / \sqrt{R} \gamma_{s} \omega_{p} \nu_{m}$, where $\gamma_{s}$ is the relativistic factor associated with the stream 
velocity of the secondary electrons and positrons. The number of wave crests in a given modulation is

$$
\mathcal{N}_{0}=\delta k / 2 \pi=\sqrt{R / 2} \pi k c / \omega_{p} \nu_{m}
$$

indicating that $\mathcal{N}_{0}$ is inversely proportional to $\nu_{m}$. This fact is observed in experiments (Cordes 1979; Ferguson 1981).

From the observed temporal pulsewidth of the pulsar microstructures $(\sim 1 \mu$ $\mathrm{s}$ to $1 \mathrm{~ms})$ and the frequency range of pulsar radio emissions $(\sim 100 \mathrm{MHz}$ to 1 $\mathrm{GHz}$ ), Chian \& Kennel (1983) noticed that $\mathcal{N}_{0}$ varies roughly from one hundred to one million. Taking typical values $\gamma_{s}=100, \nu_{0}=10^{-2}, \omega_{c}=10^{19} s^{-1}, \omega_{p}=$ $10^{6} s^{-1}, k c / \omega_{p} \approx 100, \omega \sim 10^{8} s^{-1}$, we find from our theory that $\tau_{0} \approx 1 \mu s$ and $\mathcal{N}_{0} \approx 10^{4}$. The latter is consistent with the observations (Bartel et al. 1980; Ferguson 1981).

\subsection{Amplitude modulation of circularly polarized pulsar radiation}

The evolution of the modulated circularly polarized (CP) pulsar packet in the WKB approximation is given by (Stenflo et al. 1985; Shukla 1993).

$$
i\left(\partial_{\tau}+v_{g z} \partial_{z}\right) E+\frac{1}{2} v_{g z}^{\prime} \partial_{z}^{2}-\Delta E=0,
$$

where $v_{g z}=\partial \omega / \partial k$ is the group velocity along the $\mathrm{z}$ axis, $v_{g z}^{\prime}=\partial^{2} \omega / \partial k^{2}$ is the group velocity dispersion, and the nonlinear frequency shift is given by

$$
\Delta=\frac{v_{g z}}{k c^{2}}\left[\frac{\omega^{2} \omega_{p}^{2}}{\omega^{2}-\omega_{c}^{2}} \frac{n_{1}}{n_{0}}+k V_{z} \frac{2 \omega \omega_{p}^{2} \omega_{c}^{2}}{\left(\omega^{2}-\omega_{c}^{2}\right)^{2}}+\frac{2 \omega^{2} \omega_{p}^{2} \omega_{c}^{2}}{\left(\omega^{2}-\omega_{c}^{2}\right)^{2}} \frac{B_{1 z}}{B_{0}}\right]+\Delta_{r},
$$

where $n_{1}, V_{z}$ and $B_{1 z}$ are, respectively, the density, the fluid velocity, and the compressional magnetic field perturbations associated with the plasma slow motion. The nonlinear frequency shift due to the relativistic particle mass variation is

$$
\Delta_{r}=-\frac{v_{g z}}{k c^{2}} \frac{\omega^{2}}{c^{2}} \frac{\omega_{p}^{2}\left[4 \omega^{2} \omega_{c}^{2}+\left(\omega^{2}+\omega_{c}^{2}\right)^{2}\right]}{\left(\omega^{2}-\omega_{c}^{2}\right)^{4}} \frac{e^{2}|E|^{2}}{m_{0}^{2}} .
$$

For quasi-stationary $\left(V_{z} \approx 0\right)$ modulations, we have

$$
\frac{n_{1}}{n_{0}}=\left(N^{2}-1\right) \frac{|E|^{2}}{4 \pi n_{0} T} \equiv-\frac{2 \omega_{p}^{2}}{\omega^{2}-\omega_{c}^{2}} \frac{|E|^{2}}{4 \pi n_{0} T},
$$

and

$$
\frac{B_{1 z}}{B_{0}}=-\frac{4 \omega_{p}^{2}}{\left(\omega^{2}-\omega_{c}^{2}\right)^{2}} \frac{e^{2}|E|^{2}}{m_{0}^{2} c^{2}},
$$

where $T$ is s,um of the electron and positron temperatures.

Hence, the nonlinear frequency shift takes the form

$$
\Delta=-\frac{2 v_{g z}}{k c^{2}} \frac{\omega^{2} \omega_{p}^{4}}{\left(\omega^{2}-\omega_{c}^{2}\right)^{2}}\left[\frac{|E|^{2}}{4 \pi n_{0} T}+\frac{4 \omega_{c}^{2}}{\left(\omega^{2}-\omega_{c}^{2}\right)^{2}} \frac{e^{2}|E|^{2}}{m_{0}^{2} c^{2}}\right]+\Delta_{r} \equiv-C_{m}|E|^{2} .
$$


Several comments are in order. Since $\Delta<0$, it appears that long and short wavelength fast modes are modulationally unstable as $v_{g z}^{\prime}$ is positive. We will thus have an envelope CP pulsar soliton consisting of bell shaped electric fields accompanied by compressional (rarefactional) density (magnetic field) perturbations for $\omega<\omega_{c}$. On the other hand, long wavelength slow modes remain modulationally stable because $v_{g z}^{\prime}<0$. However, the modulationally stable wave packet can propagate as a dark envelope soliton.

\section{Conclusions}

In this paper, we have shown that nonlinear effects associated with the relativistic particle mass variation, the harmonic generation, as well as the ponderomotive force can cause amplitude modulation of pulsar radiation in a strongly magnetized electron-positron plasma. The evolution of the modulated wave packet is governed by a Schrödinger-like equation. The latter describes the modulational instability and the localization of the pulsar radiation. Our results show that $\mathrm{O}$-mode pulsar radiation is always modulationally unstable, leading to the formation of envelope soliton structures. The number of wave crests in a given modulation is inversely proportional to the square root of the intensity of the pulsar radiation; a fact which is observed experimentally. On the other hand, circularly polarized fast pulsar radiation along the ambient magnetic field is subjected to a modulational instability. A possible final state of the latter is a bright envelope soliton consisting of a bell shaped electric field profile of the pulsar radiation accompanied with compressinal (rarefactional) density (magnetic field) perturbations that are driven by the ponderomotive force of the pulsar radiation. This indicates that the pulsar microstructure can be partly understood in terms of the present nonlinear model equations.

\section{References}

Arons J., Barnard J. J., 1986, ApJ, 302, 120

Asseo E., Pelleiter G., Sol H., 1990, MNRAS, 247, 529

Bartel N., Sieber W. , Graham D. A., 1980, A\&A, 87, 282

Chian A. C. L., Kennel C. F., 1983, Astrophys. Space Sci, 97,9

Cordes J. M., 1979, Space Sci. Rev, 24, 567

Ferguson D. C., 1981, In: Pulsars, IAU Symp. No. 95, eds. Sieber W., Wielebinski R., Reidel, Dordrecht, p. 141

Michel F. C., 1982, Rev. Mod. Phys., 54, 1

Ruderman M. A., Sutherland P. G., 1975, ApJ, 196, 51

Shukla P. K., 1985, Astrophys. Space Sci, 114, 381

Shukla P. K., 1993, Europhys. Lett., 22, 695

Stenflo L, Shukla P. K., Yu M. Y., 1985, Astrophys. Space Sci, 117, 303.

Sturrock P. A., 1971, ApJ, 164, 529 